\title{
Review
}

\section{Neuronal caspase-3 signaling: not only cell death}

\author{
M D'Amelio ${ }^{*, 1,2}$, V Cavallucci ${ }^{1,2}$ and F Cecconi ${ }^{*, 1,2}$
}

\begin{abstract}
Caspases are a family of cysteinyl aspartate-specific proteases that are highly conserved in multicellular organisms and function as central regulators of apoptosis. A member of this family, caspase-3, has been identified as a key mediator of apoptosis in neuronal cells. Recent studies in snail, fly and rat suggest that caspase-3 also functions as a regulatory molecule in neurogenesis and synaptic activity. In this study, in addition to providing an overview of the mechanism of caspase-3 activation, we review genetic and pharmacological studies of apoptotic and nonapoptotic functions of caspase-3 and discuss the regulatory mechanism of caspase-3 for executing nonapoptotic functions in the central nervous system. Knowledge of biochemical pathway(s) for nonapoptotic activation and modulation of caspase-3 has potential implications for the understanding of synaptic failure in the pathophysiology of neurological disorders. Fine-tuning of caspase-3 lays down a new challenge in identifying pharmacological avenues for treatment of many neurological disorders.
\end{abstract}

Cell Death and Differentiation (2010) 17, 1104-1114; do:10.1038/cdd.2009.180; published online 4 December 2009

The role of caspases in programmed cell death has been reviewed many times. ${ }^{1-3}$ It is widely accepted that, in mammals, two main pathways (Figure 1) have evolved for activating the caspase cascade, namely, the mitochondrial pathway (intrinsic pathway) and the death receptor pathway (extrinsic pathway). ${ }^{4,5}$ In the intrinsic pathway, one of the major events is mitochondrial outer membrane permeabilization (MOMP). The major role in MOMP seems to be carried out by $\mathrm{Bcl}-2$ family members. These proteins are characterized by the presence of one or more $\mathrm{Bcl}-2$ homology $(\mathrm{BH})$ domains (Figure 2) and are divided into pro- (Bax, Bak, etc.) and antiapoptotic (Bcl-2, Bcl-XL, etc.) proteins. Proapoptotic members are further divided into two subgroups: the $\mathrm{BH}$ only proteins and the proteins that possess $\mathrm{BH} 1, \mathrm{BH} 2$ and $\mathrm{BH} 3$ domains. Bax and Bak, as a consequence of apoptotic stimuli, are subjected to conformational changes and oligomerization, ${ }^{6}$ and they cause MOMP by destabilizing the lipid bilayer, creating pores or interacting with channels. ${ }^{7}$ Bax and Bak are counteracted by $\mathrm{Bcl}-2$ antiapoptotic members, which are present in the outer membrane. In this regulation, $\mathrm{BH} 3-$ only proteins have an important role. $^{8}$ The result of MOMP is the release of cytochrome $c$ into the cytoplasm, which (in the presence of dATP) induces the formation of the Apaf1-containing macromolecular complex called the apoptosome. This complex, in turn, binds and activates procaspase- 9 . Mature caspase- 9 remains bound to the apoptosome, recruiting and activating executioner caspase-3 and/or caspase-7. The activation of caspase- 3 and -7 is antagonized by IAPs (among these, XIAP is the only direct caspase-3 inhibitor), which in turn can be inhibited by Smac/Diablo and Omi/HtrA2, which are released from the intermembrane space when mitochondria are damaged.

The extrinsic pathway involves signaling from a cell surface receptor. The best-studied death receptor is Fas; Fas ligand (FasL) binding leads to receptor trimerization and recruitment of specific adaptor proteins. The Fas receptor contains a death domain (DD) in its cytoplasmic region that interacts with the adaptor protein, Fas-associated DD protein (FADD), forming a death receptor-induced signaling complex (DISC). Similar to DD, FADD contains a death effector domain (DED) and this recruits the DED-containing procaspase- 8 into the DISC. Procaspase- 8 is proteolytically activated to the enzymatically active caspase-8, which in turn activates downstream effector caspases. In some cell types, the death receptor and mitochondrion-associated death pathways are not mutually exclusive and may interact with each other. ${ }^{9}$ In this case, the mitochondrial pathway is engaged through caspase-8-mediated cleavage of Bid, a proapoptotic member of the Bcl-2 family. Once cleaved, truncated Bid (t-Bid) translocates to the mitochondria, where it induces cytochrome $c$ release, a crucial step in the mitochondrial pathway, as described above. Both the extrinsic and intrinsic pathways converge at caspase-3, which, together with other effector caspases (such as caspase-7 and -6 ), orchestrates the dismantling of diverse cell structures through cleavage of specific substrate. These caspasemediated cleavages produce the phenotypic changes observed in the apoptotic cell; some examples are shown in Figure 1.

\footnotetext{
1Dulbecco Telethon Institute at the Department of Biology, University of Rome 'Tor Vergata', Rome, Italy and 'Laboratory of Molecular Neuroembryology, IRCCS Fondazione Santa Lucia, Rome, Italy

${ }^{*}$ Corresponding authors: M D'Amelio or F Cecconi, Laboratory of Molecular Neuroembryology, IRCCS Fondazione Santa Lucia, Via del Fosso di Fiorano, 64, Rome 00143, Italy. Tel: + 39065017 03093; Fax: + 3906725 94222; E-mails: m.damelio@ hsantalucia.it or francesco.cecconi@ uniroma2.it

Keywords: apoptosis; neurodevelopment; neuron differentiation; synaptic plasticity; neurodegeneration

Abbreviations: MOMP, mitochondrial outer membrane permeabilization; BH, Bcl-2 homology; DD, death domain; FADD, Fas-associated death domain protein; DISC, death receptor-induced signaling complex

Received 31.7.09; revised 21.10.09; accepted 22.10.09; Edited by SJ Martin; published online 04.12 .09
} 


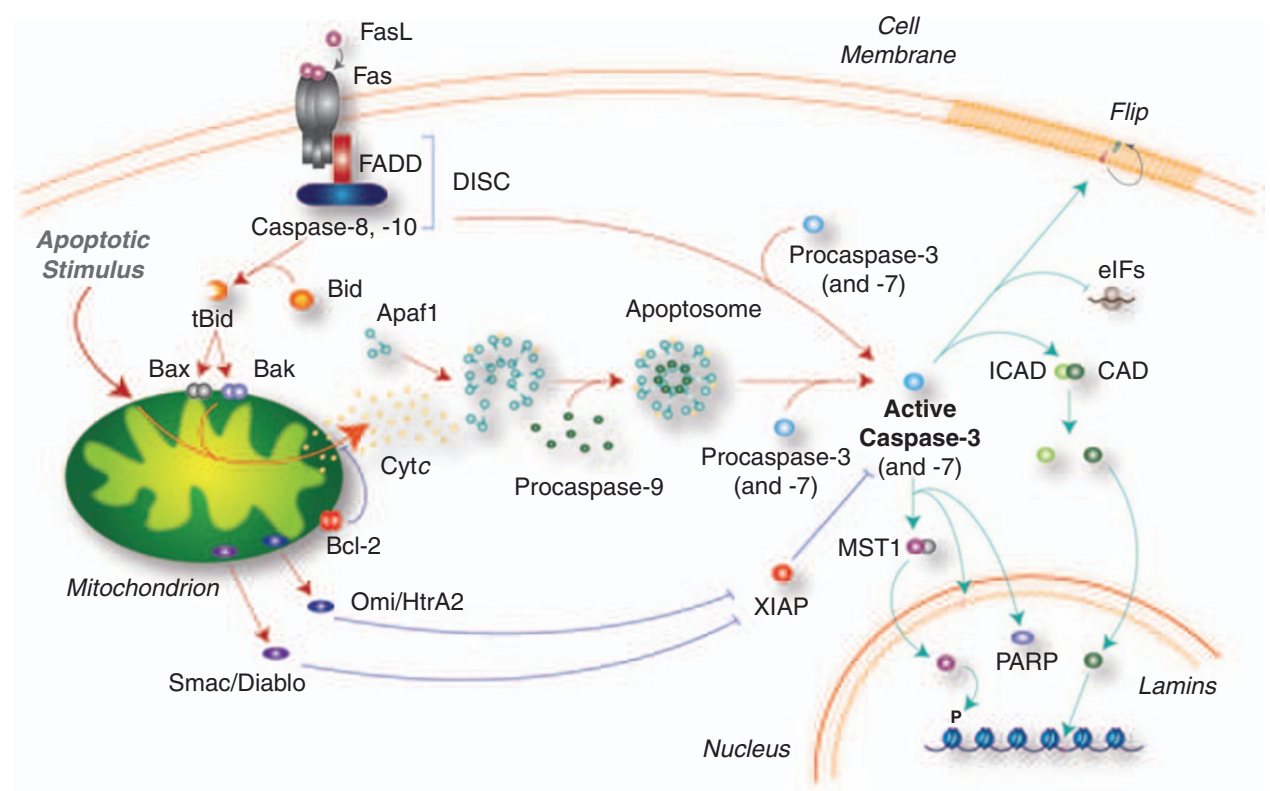

Figure 1 Intrinsic and extrinsic pathways of caspase activation in mammals. Activation of executioner caspases-3 and -7 is the key event in mammalian apoptosis, and two major mechanisms exist to carry out this task (see also text). The intrinsic pathway involves the mitochondrion, which acts as an intracellular death receptor receiving a variety of proapoptotic signals that trigger oligomerization of proapoptotic proteins (Bcl-2-associated protein, Bax, and Bcl-2-antagonist killer, Bak, to produce mitochondrial outer membrane permeabilization, MOMP). This leads to the release of cytochrome $c$, which activates Apaf1, induction of apoptosome formation, procaspase-9 recruitment/ activation and direct processing and activation of procaspase-3 and -7. In the extrinsic pathway, Fas receptor ligand (FasL) triggers the membrane-bound Death-Inducing Signaling Complex (DISC), which recruits procasapase-8 and activates caspase-3 directly. In some cell types, caspase-8 can also cleave Bid to form tBid, which interacts with Bax/Bak to trigger MOMP, cytochrome $c$ release and apoptosome formation. The activation of caspase-3 and -7 is antagonized by IAPs, which in turn can be inhibited by Smac/Diablo and Omi/HtrA2. Activation of caspase-3 and -7 orchestrates the demolition of the cell by cleavage of specific substrates, such as ICAD, ${ }^{82}$ Rho effector ROCK1 ${ }^{83}$ kinase MST1, ${ }^{84}$ PARP, ${ }^{85}$ transcription and translation initiation factors ${ }^{86}$

The caspase-dependent cell death machinery is conserved throughout evolution, from Caenorhabditis elegans to Drosophila melanogaster, and finally to mammals ${ }^{10}$ (Figure 3).

The mitochondrial pathway of mammals is essentially homologous to the programmed cell death of $C$. elegans, except that in mammals there is the essential requirement for cytochrome $c$. In $C$. elegans, the inactive CED-3 zymogen (caspases) requires activation by binding to CED-4 (Apaf1), which is bound as an inactive dimer to the mitochondrial membrane by CED-9 (Bcl-2). During cell death, EGL-1 (BH3domain protein) is upregulated and binds to CED-9, displacing the CED-4 dimer, which translocates to the perinuclear membrane, where it oligomerizes and recruits proCED-3, thus forming an apoptosome leading to CED-3 activation, which causes cell demise. ${ }^{11}$ Cytochrome $c$ does not seem to be necessary for caspase activation; indeed, CED-4 does not possess the WD-40 domain, which is a characteristic mammalian Apaf1 domain necessary for cytochrome $c$ binding. Moreover, although CED-9 binds to the mitochondria, as $\mathrm{Bcl}-2$ does, Bcl-2 acts by inhibiting MOMP, whereas in worms, MOMP is not involved in apoptosis. Nevertheless, recent studies ${ }^{12}$ suggest that mitochondria might be involved in $C$. elegans apoptosis, as they undergo fragmentation during cell death, and mitochondrial fission might be important for apoptosis regulation. However, mitochondrial remodeling in $C$. elegans was not found to be associated with MOMP and cytochrome $c$ release.
In Drosophila, Dark (also named Hac-1 or Dapaf1) promotes the activation of the initiator caspase Dronc (caspase-9); this activation is possibly regulated by the multidomain Bcl-2 family members, Debcl and Buffy. Although Dark possesses WD repeats, it seems that cytochrome $c$ is not necessary for Drosophila apoptosome formation. ${ }^{13}$ Once activated, Dronc cleaves and activates executioner caspases Drice and Dcp-1. DIAP1, which is homologous to mammalian IAP proteins, has a key role in regulating Drosophila apoptosis. DIAP1 binds processed Dronc and inhibits Dronc-Dark interaction. DIAP1 can also bind Dronc and lead to its degradation. Apoptosis is induced by upregulation of DIAP1 antagonists (Grim, HID and Reaper). Grim, HID and Reaper allow Dronc activation by causing DIAP1 autoubiquitination and degradation. ${ }^{14}$ Similar to the scenario outlined above in mammalian cells, effector caspases represent the 'point-of-no-return' in the apoptotic process, and caspase- 3 has a central role in the execution of cell death.

However, recent studies have also pointed out the importance of the nonapoptotic function of caspase-3. Spermatid individualization is an example of caspase-3 activation unrelated to cell death. ${ }^{15}$ During sperm differentiation in Drosophila, spermatids of each cyst are connected by cytoplasmic bridges, and the removal of these cytoplasmic connections is necessary to form the individual sperm, a process known as individualization. The authors found that, during this process, caspase- 3 is detected in 

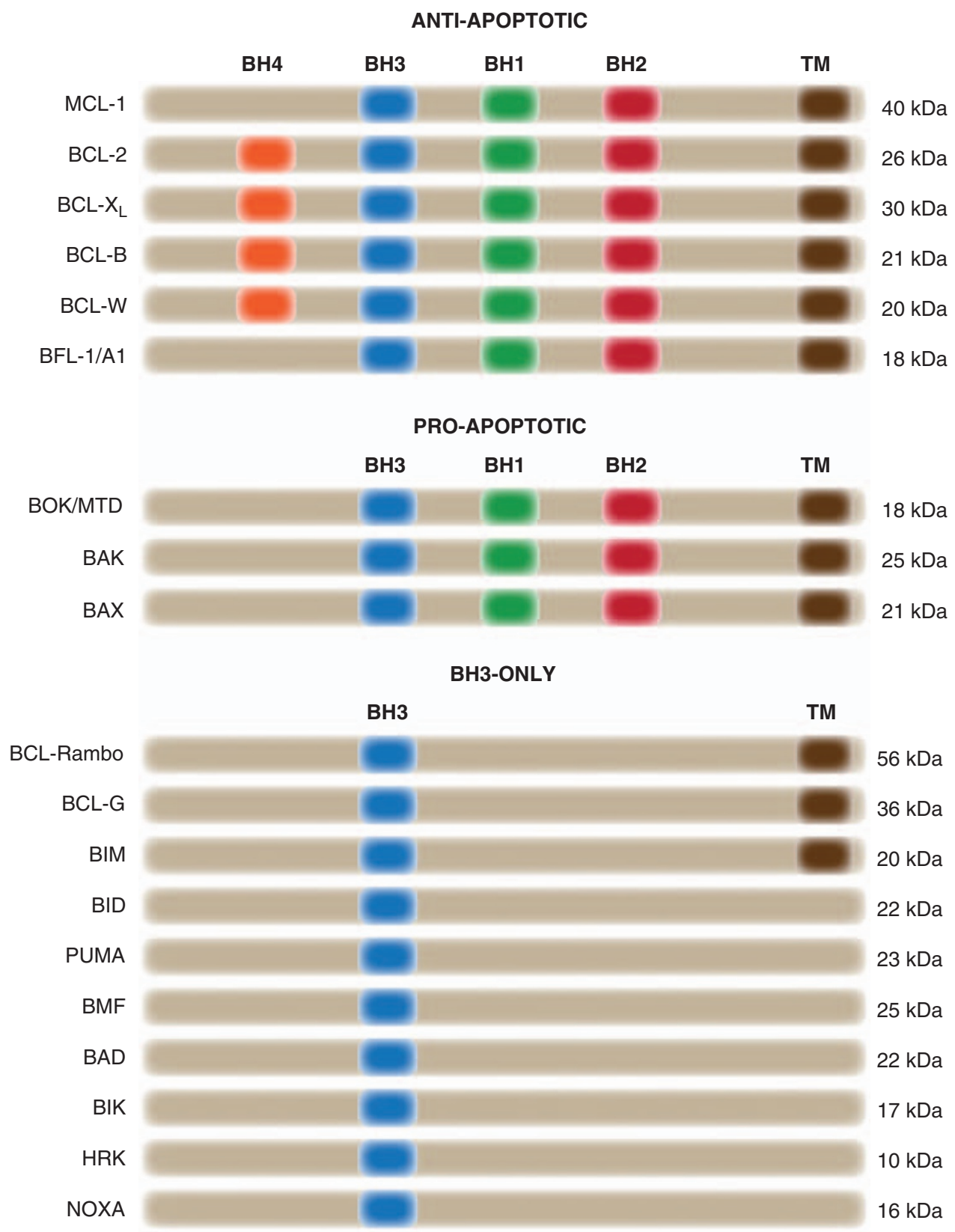

Figure 2 The Bcl-2 family. The Bcl-2 family comprises three subfamilies that contain between one and four Bcl-2 homology (BH) domains. The antiapoptotic subfamily comprises proteins that contain four BH domains. Most members of this subfamily also contain transmembrane domains (TM) and are therefore typically associated with membranes. The proapoptotic BAX-like subfamily lacks the BH4 domain. The BH3-only subfamily is a structurally diverse group of proteins, which only show homology within the small BH3 motif

the individualization complex (IC), a cytoskeletal membrane complex that moves along the length of the cyst to the sperm tail. Interestingly, Dark-dependent activation of Dronc is detected in the IC, and Dark and Dronc mutants fail to complete individualization. ${ }^{16}$

Recent developments, both in caspase research and in neurophysiology, indicate that caspase-3 is important not only in apoptosis but also in physiological processes that do not cause cell death (Figure 4). In this study, we review the apoptotic role and recent findings on the nonapoptotic functions of neuronal caspase-3, both in physiological and pathological conditions.

\section{Caspase-3 in Neurodevelopment and Differentiation}

Cell death in the developing nervous system is a phenomenon that has been recognized for almost a 100 years. It is estimated that half of the original cell population produced during nervous system development is eliminated by apoptosis to optimize synaptic connections and remove unnecessary 


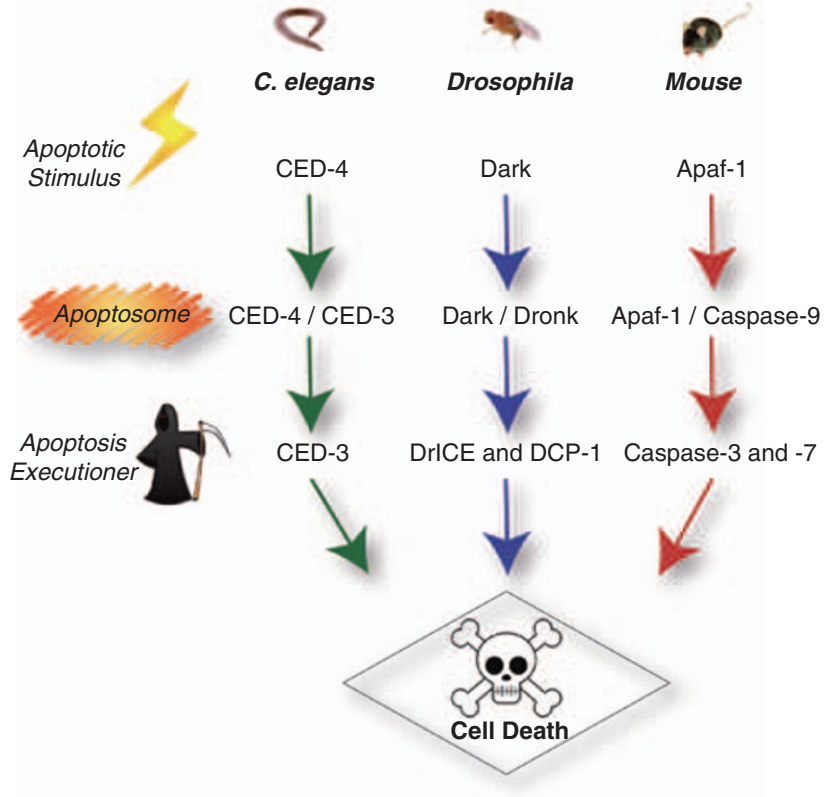

Figure 3 Conservation of the caspase-dependent cell death machinery. Apaf1 and caspases are well conserved throughout evolution (see also text). In C. elegans, the inactive CED-3 zymogen (caspases) requires activation by binding to CED-4 (Apaf1). During cell death, CED-4 translocates to the perinuclear membrane, where it oligomerizes and recruits proCED-3 forming an apoptosome, which activates CED-3, which causes cell death. In Drosophila, Dark (Apaf1) promotes the activation of the initiator caspase Dronc (caspase-9). Once activated, Dronc cleaves and activates the executioner caspases Drice and Dcp-1

neurons. In the developing nervous system, apoptosis is observed early in neural tube formation and persists during terminal differentiation of the neural network involving neuron, glial and neural progenitor cells. ${ }^{17,18}$ With a few notable exceptions, ${ }^{19,20}$ it seems that neurons predominantly use the intrinsic pathway of apoptosis to undergo cell death and that the central role of caspase- 3 in neurodevelopment is highlighted by abnormal brain development in knockout mice with an altered caspase-3 activation.

Caspase-3-deficient mice (CPP $32^{-1-}$ ) were generated by Kuida et al. ${ }^{21}$ Caspase-3 deficiency causes important defects of apoptosis in the nervous system and the majority of CPP $32^{-/-}$mice die during embryonic development or between 1 and 3 weeks of age. Development of the nervous system is strongly affected in CPP32 ${ }^{-1-}$ mice that show, at postnatal day 16 (P16), prominent protrusions of brain tissue associated with skull defects, multiple indentations of the cortex, protrusions of the neuroepithelium in the retina, accumulation of supernumerary cells in the cerebellum and ectopic cell masses sited between the cerebral cortex, the hippocampus and the striatum. At embryonic day 11.5 (E11.5), CPP32 ${ }^{-1-}$ embryos show evident brain aberrations caused by cell number increase, and the lateral, third and fourth ventricles appear abnormally narrowed. A general reduction of pyknotic cells in mutant embryos indicates that the CPP32 ${ }^{-l-}$ phenotype is principally caused by apoptosis decrease. At E16.5, the reduced apoptosis in CPP32 $2^{-1-}$ embryo brain is reflected in thicker and disorganized germinal zones, and in ventricles obstruction (Figure 5).
The phenotype of CPP32 $2^{-1-}$ mice indicates that caspase3-dependent apoptosis has an important role in the development of the nervous system. Nevertheless, the neurodevelopmental abnormalities in caspase-3-deficient mice are strain dependent, indicating that genetic factors can change the effect of caspase- 3 on brain development. ${ }^{22}$

As described above, caspase- 3 is present in the cell as an inactive proenzyme, which is cleaved and activated by caspase-9. Caspase-9-deficient mice $\left(\operatorname{Casp}^{-1-}\right)$ have been generated autonomously by two groups. ${ }^{23,24}$ Caspase- 9 loss results in perinatal lethality and in a dramatic malformation of the brain. Neural tube closure defect is evident in the hindbrain region of E10.5 $\mathrm{Casp9}^{-/-}$embryos and, at a histological level, mutants show a prominent hyperplasia of the forebrain and midbrain progenitor population with ventricular stenosis. At E13.5, the hindbrain neural tube remains open and the Casp9 $^{-/-}$brain shows an expansion of the proliferative population and the obstruction of both the lateral and third ventricle (Figure 5). By E16.5, the Casp $^{-1-}$ brain shows strong alterations, with an important expansion and protrusion (exencephaly) of cranial tissue. Casp ${ }^{-1-}$ embryos show a reduction in apoptosis during early brain development, the observed brain malformation being due to this cell death failure. It is noteworthy that Casp $^{-1-}$ embryos fail to activate caspase-3 and show a similar neuronal phenotype to that of caspase-3-deficient mice, with important brain malformations caused by an apoptosis decrease in proliferative neuroepithelium.

Caspase-9, the upstream activator of caspase-3, is activated as a consequence of cytochrome $c$ release from mitochondria and apoptosome formation. Apaf1 is the core molecule of the apoptosome, and Apaf1-deficient mice $\left(\right.$ Apaf $\left.^{-/-}\right)$have been generated. ${ }^{25}$ Apaf1 deficiency is lethal around stage $\mathrm{E} 16.5$, and $A p a f 1^{-1-}$ embryos show an abnormal development beginning at E12.5. The brain of Apaf1 $^{-1-}$ embryos, at E12.5, presents evident morphological alterations, such as overgrowth of the diencephalon and midbrain, abnormal folding and reduced size of telencephalic vesicles because of excessive cells occluding the intaventricular space (Figure 5). At E16.5, Apaf1 $^{-/}$embryos have a strong craniofacial alteration, with evident exencephaly, midline facial cleft and skull defects. Apaf1 $1^{-1-}$ embryos, similar to Casp9 $^{-1-}$ embryos, fail to activate caspase-3 and show a similar neuronal phenotype. These observations indicate a linear activation cascade from the apoptosome (containing Apaf1 and caspase-9) to caspase-3 in developing brain and highlight the importance of caspase-3 in normal neurodevelopment.

The apoptotic cascade, which leads to caspase-3 activation by apoptosome formation, starts with cytochrome $c$ release from the mitochondria to the cytosol, where it interacts with Apaf1. The role of cytochrome $c$-mediated apoptosis during neurodevelopment has been analyzed by Hao et al. ${ }^{26}$ who generated a cytochrome $c$ knock-in mouse in which the proapoptotic function of cytochrome $c$ is disrupted, although its electron transport function is intact. In this mouse model, the normal allele is replaced by a cytochrome $c$ carrying the mutation of lysine 72 to alanine (KA allele), having a reduced ability to interact with Apaf1. In fact, Lys72 is essential for the 

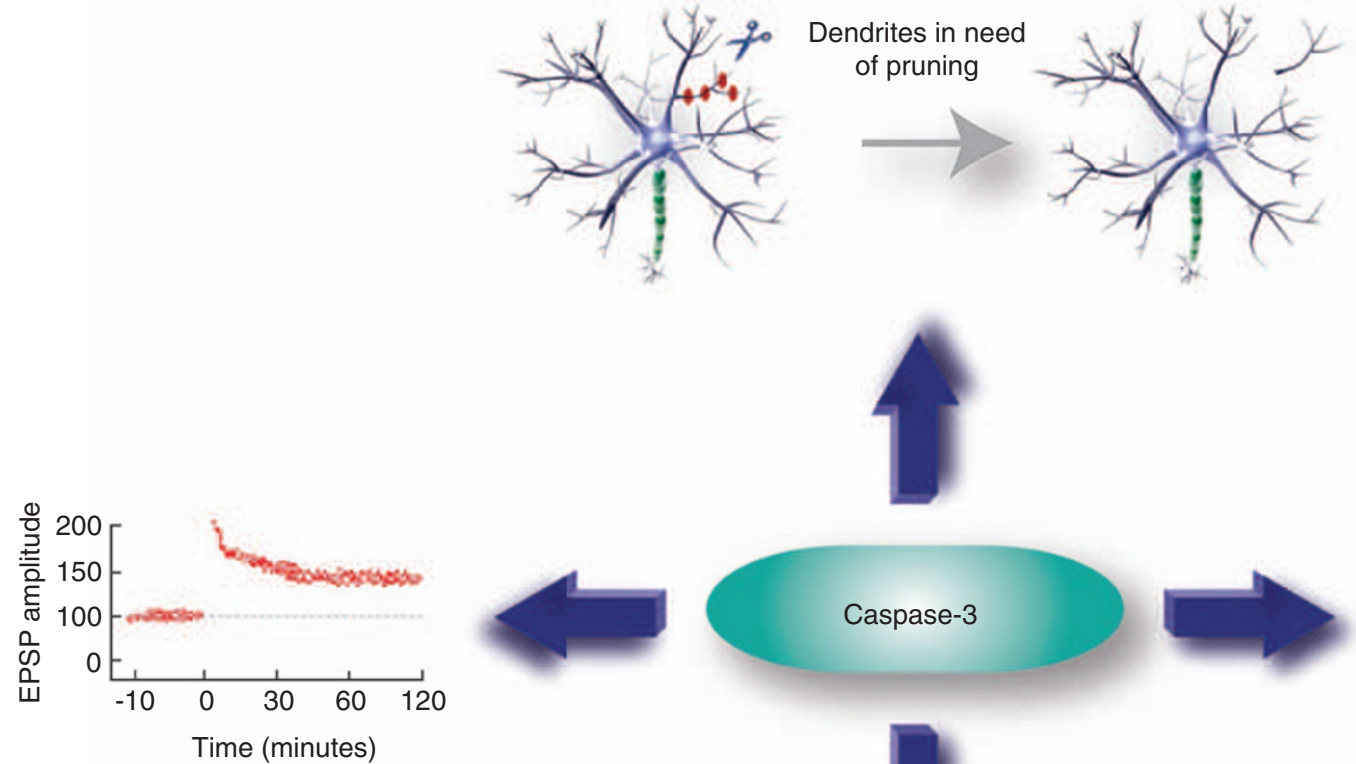

Synaptic plasticity
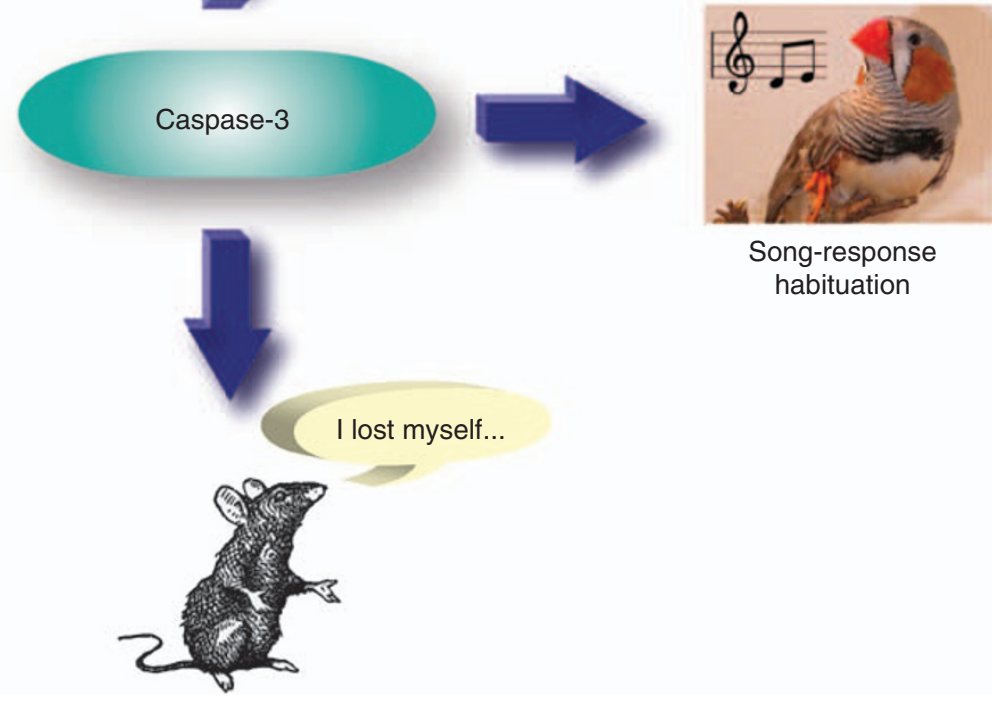

Learning and memory processes

Figure 4 Nonapoptotic caspase-3 functions in neuronal cells. Caspase-3 activation mediates nonapoptotic neuronal functions, including synaptic plasticity, dendrite pruning, as well as learning and memory processes

stability of the interaction between cytochrome $c$ and Apaf1, and the K72A mutation abolishes apoptosome formation. The KA homozygous mutation causes perinatal lethality and an abnormal brain development. At E14.5, the brain of KA/KA embryos shows ectopic masses with exencephalic defects, such as expansions of the cortex and midbrain, which cause cranial enlargement. The overgrowth of cortical neural tissue with obliteration of the ventricular space is evident in histological sections (Figure 5). The exencephalic phenotype of KA/KA mice is strictly similar to that of $\mathrm{Apaf1}^{-1-}$ and Casp $^{-/-}$mice, confirming the fundamental role of apoptotic mitochondrial pathway and caspase- 3 activation during brain development.

Although the above-mentioned deficient mice show a defective neuronal apoptosis and a dramatic brain phenotype, Apaf1-deficient mice show more profound malformations than those observed in other mutants. This observation suggests that Apaf1 may be involved in apoptotic pathways other than those involving caspase- 3 or caspase- 9 activation. Mutation of Apaf1, but not of caspase-3 or caspase-9, results in a delay in the removal of embryonic interdigital webs and also in abnormal eye development. Furthermore, as in the case of caspase-9 deficiency, Apaf1 ${ }^{-/}$cells are resistant to a wide variety of apoptotic stimuli, but not to death receptor-mediated killing. Cytochrome $c$ knock-in embryonic cells are more resistant to apoptosis induced by UV irradiation, serum starvation or staurosporin.

Last but not the least, it should be mentioned that Apaf1 also has an apoptosis-unrelated function. ${ }^{27}$ Therefore, deletion of the Apaf1 gene may lead to a phenotype different from that involving only caspase activation.

Finally, the observation that caspase-3, caspase-9 and Apaf1-deficient mice exhibit a variably severe brain phenotype, which is also likely to be strain dependent, suggests that caspase-independent death pathways may also influence nervous system development and may provide an alternative mechanism for regulating neuronal death. ${ }^{28,29}$

Caspase-3 activation is normally considered as one of the last steps in cell death. Although caspase-3 is a key protein in apoptosis execution, evidence also indicates a possible nonapoptotic role for this enzyme.

In the context of a nonapoptotic role of caspase-3, Fernando et $a l^{30}$ suggest that it is involved in neural stem cell differentiation and not only in cell death. In particular, 

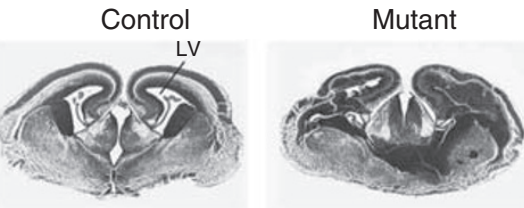

E16.5
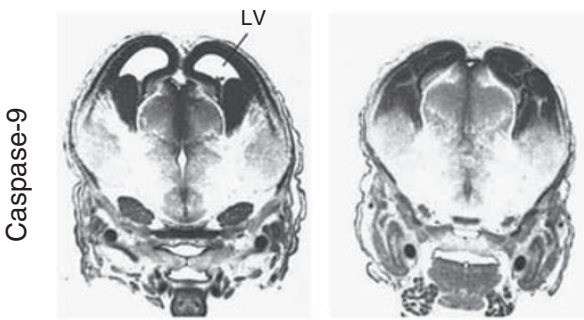

E13.5

LV
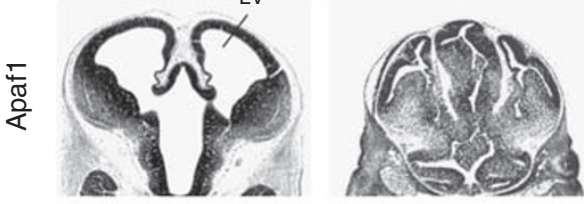

E12.5
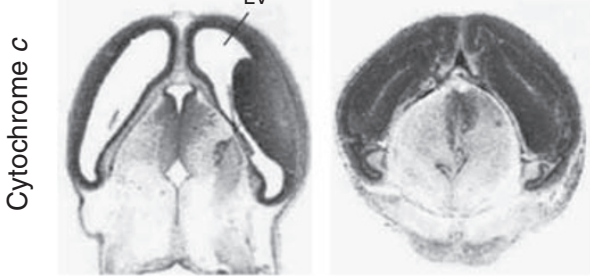

E14.5

Figure 5 Histological analysis related to the gene targeting of the caspase-3 activation component. Histological analysis of the neural embryonic phenotype of caspase-3, caspase-9, Apaf1 knockout and cytochrome $c$ knock-in mice (excerpt from Kuida et al., ${ }^{21}$ Kuida et al., ${ }^{24}$ Cecconi et al., ${ }^{87}$ and Hao et al.., ${ }^{26}$ respectively). Coronal brain sections of mutant embryos and stage-matched control are shown. Lateral ventricles (LV) in control embryos appear as open space, whereas the cortex and midbrain overexpansion in mutant embryos causes ventricular occlusion

using an in vitro system (primary derived neuronal stem cells), they showed that caspase-3 activity in neuronal progenitors facilitates neurogenesis. They generated clonally derived neurospheres from the striatum of E14.5 murine embryos and observed a significant increase in caspase- 3 activity during neurosphere differentiation. Interestingly, despite caspase-3 activity increase, no cleavage of PARP (a caspase-3 nuclear substrate cleaved during apoptosis) was observed and the caspase-3 inhibition with z-DEVD-fmk did not affect apoptosis during neurosphere differentiation, suggesting that caspase-3 activity during neurogenesis is not associated with cell death. Moreover, they showed that caspase-3 activity inhibition alters the expression of proteins associated with neurosphere differentiation and delays differentiation: (1) The nestin expression (normally expressed at a high level in early neuronal progenitors but not on differentiation) persisted under differentiation conditions in caspase-3-inhibited neurospheres; (2) GFAP and MBP expressions (which normally increase during early formation of astrocytes and oligodendrocytes, respectively) were reduced in z-DEVD-fmk-treated neurospheres compared with those in nontreated neurospheres during differentiation; (3) $\beta$-III tubulin (which normally accumulates during differentiation conditions) did not increase when caspase-3 activity was inhibited. Fernando et al. also showed that caspase-3 inhibition influences the morphology of neurospheres. The induction of differentiation is normally followed by an extension of neurite with wide networks extending from neurosphere bodies, which mimes the dendritic and axonal branching that occurs in vivo. Caspase-3 inhibition dramatically reduced neurite extension, suggesting that it is involved in morphological changes that occur during neurogenesis. Finally, the authors hypothesize that caspase-3 could promote neuronal differentiation through the activation of one or more signaling pathways by cleavage of protein kinases involved in cell differentiation.

Physiological neuronal function requires the refinement of neuronal circuits by a selective elimination of axons, dendrites and synaptic connections without the death of parental neurons. This elimination, both during development and in adult tissue, can be divided into two classes: small-scale events (elimination of synaptic connections and the local pruning of an axonal or dendritic arbor) and largescale events (elimination of a significant length of the primary axon and of major axon collaterals). ${ }^{31}$ Axonal and dendritic pruning is particularly evident in insects that undergo complete metamorphosis and build two different bodies: a larval and an adult form. During this metamorphosis, the nervous system undergoes important changes, including extensive pruning of many larval neurons that have to remodel themselves to become components of the adult nervous system. ${ }^{32}$

In this context, Williams et al. ${ }^{33}$ studied dendrite pruning in the dendritic arborizing sensory neuron ddaC of Drosophila. They observed that Dronc (the initiator caspase of fly) deficiency suppresses the pruning of ddaC dendrites and many branches persist after puparium formation; the same suppression of ddaC pruning was observed with the overexpression of a dominant-negative form of Dronc, indicating that Dronc is required for ddaC pruning. They also observed that the overexpression of either DIAP1 (the fly Dronc inhibitor) or p35 (an inhibitor of effector caspases from baculovirus without effect on Dronc) represses branch removal, indicating that effector caspases are also required in pruning. The involvement of caspases in dendritic pruning suggests that their activity is restricted to specific cellular compartments and does not cause cell death. The authors in effect proved that caspase activity is confined to the dendritic compartments of neurons during pruning and is absent in the soma or axon.

Recently, the paper from Marc Tessier-Lavigne and colleagues $^{34}$ has provided compelling evidence that Death Receptor-6 (DR6) mediates axonal pruning and degeneration induced by trophic factor withdrawal in developing neurons. The identification of DR6 as the receptor for secreted amyloid precursor protein (APP) and the link of APP to axonal degeneration through the DR6/caspase- 6 pathway are both extremely exciting findings. Even though the involvement of caspase- 6 in neurite degeneration needs to be further established both in developing neurons and in adult brain, it may provide an alternative mechanism for regulating axon pruning independently of dendritic compartments. 


\section{Caspase-3 and Neuroplasticity}

The opinion that caspase-3 is more than just a 'killer' involved in neuronal programmed cell death is supported by recent experiments carried out in snail, bird and rat, implicating caspase-3 in the regulation of synaptic plasticity. The idea of a nonapoptotic role of caspase-3 in synaptic plasticity has been formulated on the basis of two considerations: (1) many proteins that have a fundamental role in neuronal plasticity are caspase-3 substrates; ${ }^{35}$ (2) in vivo and in vitro findings indicate the involvement of caspase-3 in the molecular mechanisms of learning and memory. ${ }^{36}$

The in vivo experiments that we report below use z-DEVDfmk to inhibit caspase-3. It should be borne in mind that, although z-DEVD-fmk is the best inhibitor known for caspase3 , it also inhibits other proteases (other caspases and noncaspases) when applied in high concentrations.

At present, none of the studies have tested the involvement of caspase-3 in neuroplasticity in the mammalian system using alternative approaches (such as gene knockout or gene silencing). The reason for this could be the fact that (i) caspase-3 is a pivotal protein in neurodevelopment (knockout mice are not viable in a number of genetic backgrounds or, when viable, show behavioral abnormalities because of developmental defects), (ii) caspase-3 knockdown (by gene silencing) reduces caspase-3 expression, although its activity could be unchanged, (iii) to date, a conditional knockout mammal model is not available.

'Evidence for Synaptic Apoptosis's7 is the first study to show that apoptosis-related biochemical alterations can occur locally in synapses and dendrites of cultured neurons independently of the cell body. Mattson et al. ${ }^{37}$ showed the ability of glutamate to induce caspase-3 activation in dendrites of cultured hippocampal neurons, suggesting a role of local caspase-3 activity in synaptic and dendritic degenerative processes.

Recently, Bravarenko et al., ${ }^{38}$ using a fluorimetric approach, showed that caspase- 3 activity (DEVDase) is present in the central nervous system of the terrestrial snail, Helix (Helix lucorum). Interestingly, in electrophysiological experiments, the authors showed that blockade of caspase-3 activity, by using a z-DEVD-fmk cell-permeable inhibitor, prevented the development of the long-term stage of synaptic input sensitization, indicating that caspase-3 is essential for long-term plasticity in invertebrate neurons.

Mattson et al. ${ }^{37}$ observed dendritic localization of activated caspase-3 in cultured hippocampal neurons. However, Huesmann and Clayton ${ }^{39}$ have shown for the first time that activated caspase- 3 is present, in vivo, in the postsynaptic terminal of neurons in the auditory forebrain of zebra finch (the most common and familiar small passerine bird of central Australia). In addition, they observed that caspase- 3 activity is necessary for the development of long-term habituation to a song, as shown using caspase-3 inhibitor.

The effects of caspase-3 inhibition on different types of learning and memory were also studied in adult rats after administration of z-DEVD-fmk into cerebral ventricles. Stephanichev et al. ${ }^{40}$ showed that intracerebroventricular administration of z-DEVD-fmk decreased the number of avoidance reactions in some blocks of behavioral trials in active avoidance learning. Application of caspase-3 inhibitor z-DEVD-CHO to the cerebellar vermis stimulated the extinction of an acoustic startle reaction. These results provide strong evidence for the involvement of neuronal caspase-3 in the mechanisms of learning and memory.

At the electrophysiological level, Gulyaeva et al. ${ }^{41}$ showed that caspase-3 activity is required for long-term potentiation (LTP) in the CA1 region of rat hippocampus. The authors did not identify the precise molecular mechanism of caspase-3 involvement in LTP, although they do support the concept of the role of caspase-3 in synaptic plasticity. This result is not surprising if we consider the caspase-3 potential degradative targets in the synapse, including structural and signaling proteins. ${ }^{35}$

If caspase- 3 activity contributes to synaptic plasticity, then a mechanism must also exist for limiting its proteolytic effect at the synapse level avoiding the dismantling of the rest of the neuron. It has been proposed that synaptic caspase activity is suppressed by neurotrophic factors, cytokines and/ or caspase inhibitors that are present in the neuronal cytoplasm.

Guo and Mattson ${ }^{42}$ reported that a nine-amino acid active fragment of activity-dependent neurotrophic factor (ADNF-9) can act locally in synaptic compartments to suppress oxidative stress and preserve the function of glucose and glutamate transporters. Such synaptoprotective actions suggest roles for activity-dependent trophic signaling in preventing injury-induced synaptic pruning.

Interestingly, Huesman and Clayton ${ }^{39}$ found that, in zebra finch, BIRC4 (XIAP) coprecipitates with activated caspase-3. They interpret this result by suggesting that a pool of caspase3 is maintained in the activated state at the synapse level, and released and quickly resequestered in a complex with the BIRC4 inhibitor. In other words, the dynamic interaction between activated caspase-3 and XIAP (which inhibits caspase- 3 by binding at its active site) at the synaptic terminal makes it possible to regulate caspase- 3 activity by a mechanism for rapid release and sequestering at specific synaptic sites, thereby avoiding the proliferation of the caspase cascade to the rest of the neuron.

Recently, Arama et al. ${ }^{43}$ showed that a cullin-3-based enzyme complex (cullins are known to target proteins for degradation) is required for caspase activation during sperm differentiation in Drosophila. Their results suggest that a specific cullin-3 enzyme complex activates caspases by degrading caspase inhibitors. Such degradation may provide an alternative model for how apoptotic proteins are regulated during cellular remodeling without inducing cell death. Importantly, components of this cullin-3 enzyme complex are also required for fertility in mice and humans, indicating that this mechanism has been conserved in evolution from fruit flies to humans. Given the conserved nature of these proteins, we cannot exclude that they may have important implications for caspase regulation in other systems.

\section{Caspase-3 in Neurological Disease}

Acute and chronic neurodegenerative diseases, which include stroke, brain trauma, spinal cord injury, amyotrophic lateral sclerosis (ALS), Huntington's disease (HD), 
Alzheimer's disease (AD) and Parkinson's disease (PD), are illnesses characterized by extensive neuronal cell death. Nevertheless, increasing clinical and morphological evidence supports the concept that synapse loss, which occurs in both acute and chronic neurodegenerative diseases, might take place before, or independently of, cell death.

In this study, we summarize our current understanding of caspase-3 function in acute neurological diseases and examine the accumulating evidence of caspase-3 involvement in several neurodegenerative diseases.

Acute neurological disease. Brain ischemia is most commonly the result of the occlusion of one of the carotids or the vertebral arteries or their branches, the resulting lack of oxygen and nutrients in ischemic areas causing neuron death. Historically, cell death after ischemic injury was considered to be a necrotic type of death characterized by rapid evolution, the sudden failure of cellular energy, and swelling and rupture of organelles. ${ }^{44}$ However, substantial cell death caused by ischemia occurs over several days after injury, ${ }^{45}$ exhibiting several features of apoptosis, including chromatin condensation, DNA fragmentation, TdT-mediated dUTP nick-end labeling (TUNEL) and activation of caspases. Therefore, there is a pattern of combined necrotic and apoptotic cell death after brain ischemia and traumatic injury. Necrotic cell death occurs in the core of the infarction (where hypoxia is most severe); conversely, apoptotic cell death takes place in the ischemic penumbra (where the degree of energy/oxygen deprivation is not drastic).

Three experimental models of ischemic injury have been described: (1) the middle cerebral artery occlusion (MCAo) model of ischemia in adult rats ${ }^{46,47}$ and mice; ${ }^{47,48}$ (2) the carotid artery occlusion model of ischemia in adult rats ${ }^{49}$ and gerbils ${ }^{50}$; and (3) the model of hypoxic-ischemic brain injury in neonatal rats. ${ }^{51}$ Caspase- 3 was activated in all three models of ischemic injury.

Neurodegenerative disease. Together with acute neuronal trauma and ischemia, chronic neurodegenerative diseases are also characterized by the loss of selective neuronal populations, which often results in loss of cognitive and motor functions. Cell death in chronic neurodegenerative disorders often occurs as a result of a mutation in one or several genes, although epigenetic and environmental factors have also been involved in chronic neurodegeneration.

Neuronal death in many neurodegenerative diseases such as AD, HD, PD and ALS occurs, at least in part, by apoptosis, although this remains controversial; the main reason for this controversy is that the commonly used technique for detecting apoptosis in postmortem tissues is sometimes subject to artifacts.

Many studies have investigated the role of caspases and, in particular, of caspase-3 in neurodegenerative disease. Several proteins that are linked to neurodegenerative diseases (such as huntingtin, APP and presenilins) are also cleaved by caspase-3. On the basis of such evidence, a challenging hypothesis might be that the caspase-3-mediated cleavage of these proteins may promote the onset of neurodegenerative disease and terminally execute the terminal step of apoptosis. Below, evidence for the involvement of caspase-3 in AD, PD, HD and ALS is summarized.

Alzheimer's disease. $\mathrm{AD}$ is the most common cause of dementia. $^{52}$ The neuropathological findings of amyloid-beta $(\mathrm{A} \beta)$ plaques and tau-containing neurofibrillary tangles represent important molecular clues to the underlying pathogenesis. Genetic factors are well recognized; three rare forms of autosomal-dominant early-onset familial $A D$ have been identified and are associated with mutations in APP, presenilin 1 and presenilin 2 genes. ${ }^{53}$

Several studies have shown that neuronal loss in the cerebral cortex and hippocampus is the major cause for cognitive decline in AD, although support for apoptosis as a mechanism for neuronal death in $A D$ has been tempered in recent years. Evidence for apoptosis in $A D$ came from cell culture experiments, including exposure of neuronal cells to high concentrations of $\mathrm{A} \beta$. Using TUNEL assay, evidence of DNA fragmentation in tissue sections of the brain from $A D$ patients has been shown by several groups. ${ }^{54,55}$ In some cases, as described in the above-cited studies, TUNELpositive cells exhibited an apoptotic-like morphology, such as granulated and marginated chromatin, shrunken and irregular cell shapes and the presence of apoptotic bodies. Nevertheless, in other cases, the majority of TUNEL-positive cells showed few or none of the classical morphological features of apoptosis. ${ }^{56}$

Recent reports indicate that presenilins (presenelin 1 and 2 are linked to early-onset familial AD) are cleaved by caspase-3 in cells undergoing apoptosis. Caspase-3 also cleaves purified presenilins in vitro. ${ }^{57,58}$ The significance of presenilin cleavage by caspase in $A D$ is not known. One model proposed is that caspase-mediated cleavage of presenilins may promote the pathological generation of $\mathrm{A} \beta$. However, an alteration of the caspase cleavage site in presenilins did not affect the ability of these genes to promote $\mathrm{A} \beta$ production. $^{59}$

Tanzi and colleagues showed ${ }^{60}$ that GGA3, an adaptor protein involved in beta-site APP-cleaving enzyme (BACE) trafficking, regulates BACE levels and $\beta$-secretase activity. In addition, the authors showed that GGA3 is a substrate of caspase- 3 and the levels of GGA3 are decreased and inversely correlated with increased levels of BACE in AD. Therefore, this study suggests a novel mechanism in which caspase-3, by cleaving of GGA3, impairs the degradation and stabilization of BACE and this would then lead to increased production of the $A \beta$ peptide, thereby contributing to $A D$ pathogenesis.

Recent studies have proposed that TAR DNA-binding protein-43, TDP-43 (identified as a major disease protein in frontotemporal lobar degeneration with ubiquitin-positive inclusions, FTLD-U), may also be a substrate for caspase-3 cleavage, generating an approximate $25 \mathrm{kDa}$ fragment that has been identified in brain fractions from FTDL-U and ALS patients. ${ }^{61}$ TDP-43 is a nuclear protein that is highly conserved and ubiquitously expressed in all tissues, the function of which may be exon skipping and splicing inhibitory activity. ${ }^{62}$ Interestingly, postmortem brain section analysis indicated the presence of caspase-cleaved TDP-43 in Hirano bodies, as well as in tangles, reactive astrocytes and neuritic plaques of the AD brain. ${ }^{63}$ This result suggests 
that caspase-cleaved TDP-43 is a major finding in $A D$; however, more studies are needed to determine whether caspase-3 cleavage of TDP-43 is an early or late event, and how it may contribute to the neurodegeneration associated with this disease.

An important role for caspase- 3 in synapse degeneration is suggested by Louneva et al. ${ }^{64}$ The authors found, in both control and AD cases, a selective enrichment of caspase- 3 at synapses, particularly in the postsynaptic compartment. Moreover, AD patients exhibited significant increases in synaptic procaspase-3 and active caspase-3 expression levels compared with age-matched controls. These findings encourage to study the molecular mechanisms by which active caspase-3 might lead to progressive synaptic degeneration and ultimately to synaptic loss, the best pathological correlate of cognitive decline in AD. ${ }^{65}$

Parkinson's disease. PD is a progressive movement disorder, described in 1817 by $\mathrm{Dr}$ James Parkinson, a British physician after whom the disease is named. PD is characterized by four major clinical features: (a) rest tremor of a limb; (b) slowness of movement; (c) rigidity of the limbs or trunk; and (d) poor balance. ${ }^{66}$ Degeneration of dopaminergic neurons in the substantia nigra underlies the motor dysfunction in PD. Some studies on postmortem brain tissue of PD patients have reported the presence of apoptotic cells and DNA fragmentation in the substantia nigra. ${ }^{67,68}$ However, the significance of purely morphological human postmortem features suggestive of apoptosis has remained controversial, and the results of investigations into molecular apoptotic markers in PD brains are awaited to confirm the morphological studies. ${ }^{69}$ Recently, Hartmann et al. showed that caspase-3 is a critical factor for cell death in the substantia nigra of PD patients. ${ }^{70}$ Using caspase-3 immunohistochemistry on postmortem human brain, the authors observed (a) a positive correlation between the degree of neuronal loss in affected dopaminergic neurons in the mesencephalon of $P D$ patients and the percentage of caspase-3-positive neurons in the same neurons in control subjects; and (b) a significant decrease in caspase-3-positive pigmented neurons in the substantia nigra pars compacta of PD patients compared with controls. In other words, neurons expressing caspase- 3 are more sensitive to the pathological process, indicating that caspase- 3 is a vulnerability factor and final effector in the apoptotic death of dopaminergic neurons in PD. Interestingly, Parkin protein has recently been identified as a substrate of caspase-3. ${ }^{71}$ This result supports the hypothesis that Parkin cleavage may contribute to a vicious cycle in neurons in which caspase activation, initiated by, for example, hitherto unknown causes in sporadic PD, compromises Parkin function, hence lowering the cellular stress threshold and leading to further caspase activation.

Huntington's disease. HD, also known as Huntington's chorea or chorea major, is a neurodegenerative genetic disorder that is the most common genetic cause of repetitive abnormal movements called chorea. HD is caused by expansions of CAG repeats in the huntingtin gene resulting in polyglutamine repeats in the huntingtin protein. ${ }^{72}$ Several observations pinpoint to apoptotic neuronal death in the striatum in the course of HD. Dragunow et al. ${ }^{73}$ showed DNA strand breaks in striatal neurons. Moreover, huntingtin has been shown to be a substrate for caspase-3, the rate of cleavage increasing with the length of the huntingtin polyglutamine tract and thus providing an explanation for the gain-of-function associated with CAG expansion. Cleaved huntingtin would in turn cause additional stress on the cell, resulting in additional caspase cleavage and eventual apoptosis. The results obtained from a recently described model of polyglutamine-induced, late-onset cell degeneration in Drosophila support this hypothesis. Indeed, Warrick et al. ${ }^{74}$ showed that coexpression of p35, a caspase inhibitor, restores partial pigmentation to the eyes in which expression of polyglutamines induces loss of pigmentation and progressive degeneration of the eye.

Recently, Toulmond et al. ${ }^{75}$ showed that an inhibition of caspase-3 activity by the reversible caspase-3 inhibitor $(\mathrm{M}-826)$ results in a significant reduction in neuronal death after striatal lesions in adult rats.

Amyotrophic Lateral Sclerosis. ALS, the most common motor neuron disease in human adults is ALS, also known as Lou Gehrig's Disease. ALS is characterized by the progressive and specific loss of motor neurons in the brain, brain stem and spinal cord. ${ }^{76}$ Familial ALS-linked mutations in the copper-zinc superoxide dismutase (SOD1) gene cause motor neuron death in about $3 \%$ of ALS cases. Although the main toxic properties of familial ALS-linked mutations in SOD1 remain unclear, the terminal step in the death cascade has been established as an activation of caspase-3. Activation of caspase-3 is a central feature in cell death mediated by mutant SOD1, appearing in motor neurons ${ }^{77,78}$ and astrocytes at the time of the earliest motor neuron death in all three of the best-studied mouse models of ALS. In the mouse model SOD1 ${ }^{\text {G93A }}$, the release of cytochrome $c$ from the mitochondria is followed by activation of caspase- 9 and activation of caspase-3. ${ }^{79}$ Moreover, mice bearing a transgenic $\mathrm{Bcl}-2$ gene survive longer than other ALS mice. ${ }^{80}$ Finally, the finding of caspase-3 (and caspase-1) activation in the spinal cord of patients with ALS indicates the clinical relevance of ALS mouse models. ${ }^{79,81}$

\section{Conclusions}

Neuronal caspase-3 transduces irreversible or lifelong signaling through substrate cleavage. A strong activation of caspase-3 determines the cell fate; however, local activation, substrate specificities and its activation level all seem to be involved in many regulatory mechanisms, ranging from physiological neuron death during neurodevelopment to neuron death in neurological diseases, from synapse pruning during differentiation to synapse degeneration, and from synaptic plasticity in normal brain functioning to synaptic pathology observed in neurodegenerative disease. It is expected that, in coming years, additional caspase-3 inhibitors will become a part of the drugs that clinicians administer everyday to patients suffering from neurological diseases involving caspase-mediated neuronal dysfunction and neuronal death. 


\section{Conflict of interest}

The authors declare no conflict of interest.

Acknowledgements. We are grateful to Drs Richard A Flavell, Chia-Yi Kuan and Tak W Mak for images showing histological analyses of the neural phenotype of mutant embryos. We also thank M Acuña Villa and MW Bennett for their excellent editorial and secretarial work. Our research is supported by the Telethon Foundation, AIRC, the Italian Ministry of University and Research, Ricerca Corrente and Ricerca Finalizzata for the Italian Ministry of Health and Compagnia di San Paolo.

1. Nicholson DW, Thornberry NA. Apoptosis. Life and death decisions. Science 2003; 299 214-215.

2. Creagh EM, Martin SJ. Caspases: cellular demolition experts. Biochem Soc Trans 2001 29: 696-702

3. Earnshaw WC, Martins LM, Kaufmann SH. Mammalian caspases: structure, activation, substrates, and functions during apoptosis. Annu Rev Biochem 1999; 68: 383-424.

4. Green DR, Kroemer G. The pathophysiology of mitochondrial cell death. Science 2004; 305: 626-629.

5. Schulze-Osthoff K, Ferrari D, Los M, Wesselborg S, Peter ME. Apoptosis signaling by death receptors. Eur J Biochem 1998; 254: 439-459.

6. Er E, Oliver L, Cartron PF, Juin P, Manon S, Vallette FM. Mitochondria as the target of the pro-apoptotic protein Bax. Biochim Biophys Acta 2006; 1757: 1301-1311.

7. Kroemer G, Galluzzi L, Brenner C. Mitochondrial membrane permeabilization in cell death Physiol Rev 2007; 87: 99-163.

8. Labi V, Erlacher M, Kiessling S, Villunger A. BH3-only proteins in cell death initiation, malignant disease and anticancer therapy. Cell Death Differ 2006; 13: 1325-1338.

9. Roy S, Nicholson DW. Cross-talk in cell death signaling. J Exp Med 2000; 192: F21-F25.

10. Degterev A, Yuan J. Expansion and evolution of cell death programmes. Nat Rev Mol Cell Biol 2008; 9: 378-390.

11. Lettre G, Hengartner MO. Developmental apoptosis in C. elegans: a complex CEDnario. Nat Rev Mol Cell Biol 2006; 7: 97-108.

12. Jagasia R, Grote P, Westermann B, Conradt B. DRP-1-mediated mitochondrial fragmentation during EGL-1-induced cell death in C. elegans. Nature 2005; 433: 754-760.

13. Yu X, Wang L, Acehan D, Wang X, Akey CW. Three-dimensional structure of a double apoptosome formed by the Drosophila Apaf-1 related killer. J Mol Biol 2006; 355: 577-589.

14. Vaux DL, Silke J. IAPs - the ubiquitin connection. Cell Death Differ 2005; 12: 1205-1207.

15. Arama E, Agapite J, Steller H. Caspase activity and a specific cytochrome $\mathrm{C}$ are required for sperm differentiation in Drosophila. Dev Cell 2003; 4: 687-697.

16. Arama E, Bader M, Srivastava M, Bergmann A, Steller H. The two Drosophila cytochrome $C$ proteins can function in both respiration and caspase activation. EMBO J 2006; 25 : 232-243.

17. Burek M, Oppenheim R. Cellular interactions that regulate programmed cell death in the developing vertebrate nervous system. In: Koliatsos V, Ratan R (eds). Cell Death and Disease of the Nervous System. Humana: Totowa, 1999, pp 145-180.

18. Mazarakis ND, Edwards AD, Mehmet H. Apoptosis in neural development and disease. Arch Dis Child 1997; 77: F165-F170.

19. Raoul C, Henderson CE, Pettmann B. Programmed cell death of embryonic motoneurons triggered through the Fas death receptor. J Cell Biol 1999; 147: 1049-1062.

20. Martin-Villalba A, Herr I, Jeremias I, Hahne M, Brandt R, Vogel J et al. CD95 ligand (Fas-L/APO-1L) and tumor necrosis factor-related apoptosis-inducingligand mediate ischemia-induced apoptosis in neurons. J Neurosci 1999; 19: 3809-3817.

21. Kuida K, Zheng TS, Na S, Kuan C, Yang D, Karasuyama $\mathrm{H}$ et al. Decreased apoptosis in the brain and premature lethality in CPP32-deficient mice. Nature 1996; 384: 368-372.

22. Leonard JR, Klocke BJ, D'Sa C, Flavell RA, Roth KA. Strain-dependent neurodevelopmental abnormalities in caspase-3-deficient mice. J Neuropathol Exp Neurol 2002; 61: 673-677.

23. Hakem R, Hakem A, Duncan GS, Henderson JT, Woo M, Soengas MS et al. Differential requirement for caspase 9 in apoptotic pathways in vivo. Cell 1998; 94: 339-352.

24. Kuida K, Haydar TF, Kuan CY, Gu Y, Taya C, Karasuyama H et al. Reduced apoptosis and cytochrome c-mediated caspase activation in mice lacking caspase 9. Cell 1998; 94 325-337.

25. Cecconi F, Alvarez-Bolado G, Meyer BI, Roth KA, Gruss P. Apaf1 (CED-4 homolog) regulates programmed cell death in mammalian development. Cell 1998; 94: 727-737.

26. Hao Z, Duncan GS, Chang CC, Elia A, Fang M, Wakeham A et al. Specific ablation of the apoptotic functions of cytochrome $C$ reveals a differential requirement for cytochrome $C$ and Apaf-1 in apoptosis. Cell 2005; 121: 579-591.

27. Zermati Y, Mouhamad S, Stergiou L, Besse B, Galluzzi L, Boehrer S et al. Nonapoptotic role for Apaf-1 in the DNA damage checkpoint. Mol Cell 2007; 28: 624-637.

28. Clarke PG. Developmental cell death: morphological diversity and multiple mechanisms. Anat Embryol (Berl) 1990; 181: 195-213.
29. Zaidi AU, McDonough JS, Klocke BJ, Latham CB, Korsmeyer SJ, Flavell RA et al. Chloroquine-induced neuronal cell death is p53 and Bcl-2 family-dependent but caspaseindependent. J Neuropathol Exp Neurol 2001; 60: 937-945.

30. Fernando $P$, Brunette $S$, Megeney LA. Neural stem cell differentiation is dependent upon endogenous caspase 3 activity. FASEB J 2005; 19: 1671-1673.

31. Luo L, O'Leary DD. Axon retraction and degeneration in development and disease. Annu Rev Neurosci 2005; 28: 127-156.

32. Truman JW. Metamorphosis of the central nervous system of Drosophila. $J$ Neurobiol 1990; 21: 1072-1084

33. Williams DW, Kondo S, Krzyzanowska A, Hiromi Y, Truman JW. Local caspase activity directs engulfment of dendrites during pruning. Nat Neurosci 2006; 9: 1234-1236.

34. Nikolaev A, McLaughlin T, O'Leary DD, Tessier-Lavigne M. APP binds DR6 to trigger axon pruning and neuron death via distinct caspases. Nature 2009; 457: 981-989.

35. Chan SL, Mattson MP. Caspase and calpain substrates: roles in synaptic plasticity and cell death. J Neurosci Res 1999; 58: 167-190.

36. Dash PK, Blum S, Moore AN. Caspase activity plays an essential role in long-term memory. Neuroreport 2000; 11: 2811-2816.

37. Mattson MP, Keller JN, Begley JG. Evidence for synaptic apoptosis. Exp Neurol 1998; 153 : 35-48.

38. Bravarenko NI, Onufriev MV, Stepanichev MY, lerusalimsky VN, Balaban PM, Gulyaeva NV. Caspase-like activity is essential for long-term synaptic plasticity in the terrestrial snail Helix. Eur J Neurosci 2006; 23: 129-140.

39. Huesmann GR, Clayton DF. Dynamic role of postsynaptic caspase-3 and BIRC4 in zebra finch song-response habituation. Neuron 2006; 52: 1061-1072.

40. Stepanichev MY, Kudryashova IV, Yakovlev AA, Onufriev MV, Khaspekov LG, Lyzhin AA et al. Central administration of a caspase inhibitor impairs shuttle-box performance in rats. Neuroscience 2005; 136: 579-591.

41. Gulyaeva NV, Kudryashov IE, Kudryashova IV. Caspase activity is essential for long-term potentiation. J Neurosci Res 2003; 73: 853-864.

42. Guo ZH, Mattson MP. Neurotrophic factors protect cortical synaptic terminals against amyloid and oxidative stress-induced impairment of glucose transport, glutamate transport and mitochondrial function. Cereb Cortex 2000; 10: 50-57.

43. Arama $\mathrm{E}$, Bader $\mathrm{M}$, Rieckhof $\mathrm{GE}$, Steller $\mathrm{H}$. A ubiquitin ligase complex regulates caspase activation during sperm differentiation in Drosophila. PLOS Biol 2007; 5: e251.

44. Choi DW. Calcium and excitotoxic neuronal injury. Ann N Y Acad Sci 1994; 747: 162-171.

45. Pulsinelli WA, Brierley JB, Plum F. Temporal profile of neuronal damage in a model of transient forebrain ischemia. Ann Neurol 1982; 11: 491-498.

46. Loddick SA, MacKenzie A, Rothwell NJ. An ICE inhibitor, z-VAD-DCB attenuates ischaemic brain damage in the rat. Neuroreport 1996; 7: 1465-1468.

47. Hara H, Friedlander RM, Gagliardini V, Ayata C, Fink K, Huang Z et al. Inhibition of interleukin 1beta converting enzyme family proteases reduces ischemic and excitotoxic neuronal damage. Proc Natl Acad Sci USA 1997; 94: 2007-2012.

48. Schielke GP, Yang GY, Shivers BD, Betz AL. Reduced ischemic brain injury in interleukin-1 beta converting enzyme-deficient mice. J Cereb Blood Flow Metab 1998; 18: 180-185.

49. Chen J, Nagayama T, Jin K, Stetler RA, Zhu RL, Graham SH et al. Induction of caspase-3like protease may mediate delayed neuronal death in the hippocampus after transient cerebral ischemia. J Neurosci 1998; 18: 4914-4928.

50. Himi T, Ishizaki Y, Murota S. A caspase inhibitor blocks ischaemia-induced delayed neuronal death in the gerbil. Eur J Neurosci 1998; 10: 777-781.

51. Cheng Y, Deshmukh M, D'Costa A, Demaro JA, Gidday JM, Shah A et al. Caspase inhibitor affords neuroprotection with delayed administration in a rat model of neonatal hypoxic-ischemic brain injury. J Clin Invest 1998; 101: 1992-1999.

52. Kawas CH. Clinical practice. Early Alzheimer's disease. N Engl J Med 2003; 349: 1056-1063.

53. Selkoe DJ. Alzheimer's disease: genes, proteins, and therapy. Physiol Rev 2001; 81: 741-766.

54. Lassmann $\mathrm{H}$, Bancher $\mathrm{C}$, Breitschopf $\mathrm{H}$, Wegiel J, Bobinski M, Jellinger $\mathrm{K}$ et al. Cell death in Alzheimer's disease evaluated by DNA fragmentation in situ. Acta Neuropathol 1995; 89: 35-41.

55. Su JH, Anderson AJ, Cummings BJ, Cotman CW. Immunohistochemical evidence for apoptosis in Alzheimer's disease. Neuroreport 1994; 5: 2529-2533.

56. Smale G, Nichols NR, Brady DR, Finch CE, Horton Jr WE. Evidence for apoptotic cell death in Alzheimer's disease. Exp Neurol 1995; 133: 225-230.

57. Kim TW, Pettingell WH, Jung YK, Kovacs DM, Tanzi RE. Alternative cleavage of Alzheimer-associated presenilins during apoptosis by a caspase-3 family protease. Science 1997; 277: 373-376.

58. Loetscher $H$, Deuschle U, Brockhaus M, Reinhardt D, Nelboeck $P$, Mous $J$ et al. Presenilins are processed by caspase-type proteases. J Biol Chem 1997; 272: 20655-20659.

59. Brockhaus M, Grünberg J, Röhrig S, Loetscher H, Wittenburg N, Baumeister R et al. Caspase-mediated cleavage is not required for the activity of presenilins in amyloidogenesis and NOTCH signaling. Neuroreport 1998; 9: 1481-1486.

60. Tesco G, Koh YH, Kang EL, Cameron AN, Das S, Sena-Esteves M et al. Depletion of GGA3 stabilizes BACE and enhances beta-secretase activity. Neuron 2007; 54: 721-737.

61. Neumann M, Sampathu DM, Kwong LK, Truax AC, Micsenyi MC, Chou TT et al. Ubiquitinated TDP-43 in frontotemporal lobar degeneration and amyotrophic lateral sclerosis. Science 2006; 314: 130-133. 
62. Buratti E, Brindisi A, Pagani F, Baralle FE. Nuclear factor TDP-43 binds to the polymorphic TG repeats in CFTR intron 8 and causes skipping of exon 9: a functional link with disease penetrance. Am J Hum Genet 2004; 74: 1322-1325.

63. Rohn TT. Caspase-cleaved TAR DNA-binding protein-43 is a major pathological finding in Alzheimer's disease. Brain Res 2008; 1228: 189-198.

64. Louneva N, Cohen JW, Han LY, Talbot K, Wilson RS, Bennett DA et al. Caspase-3 is enriched in postsynaptic densities and increased in Alzheimer's disease. Am J Pathol 2008; 173: 1488-1495.

65. Scheff SW, Price DA, Schmitt FA, DeKosky ST, Mufson EJ. Synaptic alterations in CA1 in mild Alzheimer's disease and mild cognitive impairment. Neurology 2007; 68 : 1501-1508.

66. Jankovic J. Parkinson's disease: clinical features and diagnosis. J Neurol Neurosurg Psychiatry 2008; 79: 368-376.

67. Mochizuki H, Goto K, Mori H, Mizuno Y. Histochemical detection of apoptosis in Parkinson's disease. J Neurol Sci 1996; 137: 120-123.

68. Hirsch EC, Hunot S, Faucheux B, Agid Y, Mizuno Y, Mochizuki H et al. Dopaminergic neurons degenerate by apoptosis in Parkinson's disease. Mov Disord 1999; 14: 383-385.

69. Banati RB, Daniel SE, Blunt SB. Glial pathology but absence of apoptotic nigral neurons in long-standing Parkinson's disease. Mov Disord 1998; 13: 221-227.

70. Hartmann A, Hunot S, Michel PP, Muriel MP, Vyas S, Faucheux BA et al. Caspase-3: a vulnerability factor and final effector in apoptotic death of dopaminergic neurons in Parkinson's disease. Proc Natl Acad Sci USA 2000; 97: 2875-2880.

71. Kahns S, Lykkebo S, Jakobsen LD, Nielsen MS, Jensen PH. Caspase-mediated parkin cleavage in apoptotic cell death. Biol Chem 2002; 277: 15303-15308.

72. Walker FO. Huntington's disease. Lancet 2007; 369: 218-228.

73. Dragunow M, Faull RL, Lawlor P, Beilharz EJ, Singleton K, Walker EB et al. In situ evidence for DNA fragmentation in Huntington's disease striatum and Alzheimer's disease temporal lobes. Neuroreport 1995; 6: 1053-1057.

74. Warrick JM, Paulson HL, Gray-Board GL, Bui QT, Fischbeck KH, Pittman RN et al. Expanded polyglutamine protein forms nuclear inclusions and causes neural degeneration in Drosophila. Cell 1998; 93: 939-949.

75. Toulmond S, Tang K, Bureau Y, Ashdown H, Degen S, O'Donnell R et al. Neuroprotective effects of M826, a reversible caspase- 3 inhibitor, in the rat malonate model of Huntington's disease. Br J Pharmacol 2004; 141: 689-697.
76. Rowland LP, Shneider NA. Amyotrophic lateral sclerosis. N Engl J Med 2001; 344 $1688-1700$.

77. Li M, Ona VO, Guégan C, Chen M, Jackson-Lewis V, Andrews LJ et al. Functional role of caspase-1 and caspase-3 in an ALS transgenic mouse model. Science 2000; 288 335-339.

78. Pasinelli P, Houseweart MK, Brown Jr RH, Cleveland DW Caspase-1 and -3 are sequentially activated in motor neuron death in $\mathrm{Cu}, \mathrm{Zn}$ superoxide dismutasemediated familial amyotrophic lateral sclerosis. Proc Natl Acad Sci USA 2000; 97 13901-13906.

79. Guégan C, Vila M, Rosoklija G, Hays AP, Przedborski S. Recruitment of the mitochondrialdependent apoptotic pathway in amyotrophic lateral sclerosis. J Neurosci 2001; 21 6569-6576.

80. Kostic V, Jackson-Lewis V, de Bilbao F, Dubois-Dauphin M, Przedborski S. Bcl-2 prolonging life in a transgenic mouse model of familial amyotrophic lateral sclerosis. Science 1997; 277: 559-562.

81. Zhu S, Stavrovskaya IG, Drozda M, Kim BY, Ona V, Li M et al. Minocycline inhibits cytochrome $\mathrm{c}$ release and delays progression of amyotrophic lateral sclerosis in mice. Nature 2002; 417: 74-78.

82. Enari M, Sakahira H, Yokoyama H, Okawa K, Iwamatsu A, Nagata S. A caspaseactivated DNase that degrades DNA during apoptosis, and its inhibitor ICAD. Nature 1998; 391: $43-50$

83. Coleman ML, Sahai EA, Yeo M, Bosch M, Dewar A, Olson MF. Membrane blebbing during apoptosis results from caspase-mediated activation of ROCK I. Nat Cell Biol 2001; 3: 339-345.

84. Ura S, Masuyama N, Graves JD, Gotoh Y. Caspase cleavage of MST1 promotes nuclear translocation and chromatin condensation. Proc Natl Acad Sci USA 2001; 98 10148-10153.

85. Rosen A, Casciola-Rosen L. Macromolecular substrates for the ICE-like proteases during apoptosis. J Cell Biochem 1997; 64: 50-54.

86. Lüthi AU, Martin SJ. The CASBAH: a searchable database of caspase substrates. Cell Death Differ 2007; 14: 641-650.

87. Cecconi F, Piacentini M, Fimia GM. The involvement of cell death and survival in neural tube defects: a distinct role for apoptosis and autophagy? Cell Death Differ 2008; 15 $1170-1177$. 